\title{
Chronic Coinfections in Patients Diagnosed with Chronic Lyme Disease: A Systematic Review
}

\author{
Paul M. Lantos, MD, ${ }^{\mathrm{a}, \mathrm{b}}$ Gary P. Wormser, MD ${ }^{\mathrm{c}}$
}

${ }^{a}$ Division of Pediatric Infectious Diseases and ${ }^{b}$ Division of General Internal Medicine, Duke University School of Medicine, Durham, NC; ${ }^{c}$ Division of Infectious Diseases, New York Medical College, Valhalla.

\begin{abstract}
PURPOSE: Often, the controversial diagnosis of chronic Lyme disease is given to patients with prolonged, medically unexplained physical symptoms. Many such patients also are treated for chronic coinfections with Babesia, Anaplasma, or Bartonella in the absence of typical presentations, objective clinical findings, or laboratory confirmation of active infection. We have undertaken a systematic review of the literature to evaluate several aspects of this practice.

METHODS: Five systematic literature searches were performed using Boolean operators and the PubMed search engine.

RESULTS: The literature searches did not demonstrate convincing evidence of: 1) chronic anaplasmosis infection; 2) treatment-responsive symptomatic chronic babesiosis in immunocompetent persons in the absence of fever, laboratory abnormalities, and detectable parasitemia; 3 ) either geographically widespread or treatment-responsive symptomatic chronic infection with Babesia duncani in the absence of fever, laboratory abnormalities, and detectable parasitemia; 4) tick-borne transmission of Bartonella species; or 5) simultaneous Lyme disease and Bartonella infection.
\end{abstract}

CONCLUSIONS: The medical literature does not support the diagnosis of chronic, atypical tick-borne coinfections in patients with chronic, nonspecific illnesses.

(C) 2014 Elsevier Inc. All rights reserved. • The American Journal of Medicine (2014) 127, 1105-1110

KEYWORDS: Anaplasma; Babesia; Bartonella; Borrelia burgdorferi; Coinfection; Lyme disease

Lyme disease is the most commonly reported vector-borne infection in the US with over 30,000 confirmed or probable cases in 2011. ${ }^{1}$ Lyme disease is caused by infection

Funding: PML was supported by the National Center for Advancing Translational Sciences of the National Institutes of Health under award number KL2TR001115. The content is solely the responsibility of the authors and does not necessarily represent the official views of the National Institutes of Health.

Conflicts of Interest: GPW received research grants from the Centers for Disease Control and Prevention, National Institutes of Health, Immunetics, Inc., Bio-Rad, DiaSorin, Inc., and BioMerieux holds equity in Abbott. GPW was he was an expert witness in malpractice cases involving Lyme disease; an unpaid board member of the American Lyme Disease Foundation; expert witness regarding Lyme disease in a disciplinary action for the Missouri Board of Registration for the Healing Arts; and a consultant to Baxter for Lyme vaccine development. PML has no potential conflicts of interest to report.

Authorship: Both authors had access to all data and took part in writing this manuscript.

Requests for reprints should be addressed to Paul M. Lantos, MD, Division of General Internal Medicine, Duke University School of Medicine, DUMC 100800, Durham, NC 27710.

E-mail address: Paul.1antos@duke.edu with the spirochete Borrelia burgdorferi and transmitted by Ixodes spp. ticks.

While many aspects of Lyme disease are well accepted by the mainstream medical community, considerable controversy surrounds "chronic Lyme disease," an ill-defined diagnosis that some clinicians give to patients with alternative diagnoses or medically unexplained symptom complexes. In many instances these patients also are diagnosed with chronic coinfection with Anaplasma, Babesia, or Bartonella. In the context of chronic Lyme disease, these pathogens often are diagnosed in the absence of typical presentations or objective clinical findings, and without laboratory confirmation.

In this systematic review we address several major questions relevant to the diagnosis of coinfections in patients with a diagnosis of chronic Lyme disease. These questions are the following:

1) Is there evidence of persistent human granulocytic anaplasmosis (HGA)?

2) How is relapsing or persisting babesiosis identified and diagnosed? 
3) Has chronic Babesia duncani infection been described?

4) Is there convincing evidence for tick-borne human Bartonella infection?

5) Is there convincing evidence for simultaneous Lyme disease and Bartonella infection?

\section{METHODS}

In order to identify relevant articles, we performed the following Boolean searches of the indexed medical literature using the PubMed search engine.

\section{Search 1}

For evidence of chronic human anaplasmosis:

(anaplasma OR anaplasmosis OR ehrlichia OR ehrlichiosis OR phagocytophilum) AND (chronic OR persistent $O R$ recurrent $O R$ relapse)

\section{Search 2}

To characterize chronic or relapsing babesiosis:

(babesia OR babesiosis) AND (chronic OR persistent OR recurrent OR relapse)

\section{Search 3}

For the role of Babesia duncani in human disease:

babesia AND (duncani OR WA1)

\section{Search 4}

For tick-borne Bartonella infection:

(tick OR Ixodes) AND (bartonella OR bartonellosis)

\section{Search 5}

For simultaneous Lyme disease and bartonellosis:

\section{(Lyme OR borrelia OR borreliosis) AND (bartonella OR bartonellosis)}

Case reports, case series, and primary scientific studies were selected from among the search results. Review articles, correspondence, and editorials were excluded. We limited our search to studies with human subjects. This was done by manually reviewing the articles and excluding those in which the subjects were nonhuman (rather than adding a search function limit to the PubMed query). Because Anaplasma phagocytophilum was formerly categorized as Ehrlichia, we included Ehrlichia and ehrlichiosis in the search terms for this query.

\section{RESULTS}

\section{Search 1: Persistent, Chronic, or Recurrent Human Granulocytic Anaplasmosis}

This search yielded 252 articles. The vast majority of scientific articles yielded by these search terms were animal studies. Many addressed microorganisms other than A. phagocytophilum. Ultimately, only 2 studies were appropriate for further review based on our inclusion criteria. In the first, 2 febrile asplenic patients were diagnosed with HGA based on blood smear examination. ${ }^{2}$ One developed neurologic symptoms including left-sided weakness, left hemi-neglect, and delirium within 12 days of an admission in which HGA had been diagnosed and treated. His blood smear examination was negative at this second visit and he was apparently afebrile, so recurrent HGA was not definitively established; nonetheless, he received doxycycline and promptly improved. The second asplenic patient was treated uneventfully with 10 days of doxycycline and suffered no relapse. A second study reported HGA in 3 recipients of pancreas transplantation. ${ }^{3}$ While all of the patients had overall complicated medical courses, none had evidence of recurrent or chronic HGA. This study was reported from Kentucky, a state where HGA is not known to be endemic.

\section{Search 2: Persistent or Relapsing Human Babesiosis}

This search yielded 200 articles. Of these, 31 were retrieved for further analysis after screening as described in the Methods section. A large number of these studies documented relapsing or persistent babesiosis or babesiosis whose diagnosis was delayed; complicated disease predominantly affected asplenic or otherwise immunocompromised patients. Fever, laboratory abnormalities such as anemia, and direct evidence of parasitemia such as a positive blood film examination or polymerase chain reaction (PCR) assay were nearly universal among the reported patients. ${ }^{4-23}$

The literature search did not yield evidence of cryptic babesiosis resulting in a less overt syndrome. A study of patients with chronic fatigue syndrome found seroreactivity to Babesia microti in 2 controls but not in any of the study subjects with chronic fatigue. ${ }^{24} \mathrm{~A}$ case series of 3 patients attributed panic attacks to infection with multiple tick-borne pathogens including babesiosis. ${ }^{25}$ In 2 of the 3 cases, presumption of babesiosis was based solely on antibody titers - an immunoglobulin $\mathrm{M}$ (IgM) titer of 1:80 in one case and a "low positive" titer in the other. A third patient in this series reportedly had B. microti DNA detected by PCR. Details of the PCR reaction were not provided, there was no report of a blood film examination, and no report of 
laboratory testing to evaluate hemolysis; the article reports that the patient's panic attacks were eliminated after 9 months of "increasingly aggressive antimicrobial therapy for tick-borne diseases." None of the antibiotics listed in the article has known efficacy for human babesiosis.

\section{Search 3: Babesia Duncani Infection}

This search yielded 26 articles. Of these, we identified 13 case reports, case series, or human studies for further review. The remainder was comprised of review material or animal studies. Two instances were reports of a Babesia divergens-like pathogen, and infection with $B$. duncani was excluded.

Infection with $B$. duncani, formerly designated WA1, has been described in 8 patients in the medical literature. ${ }^{26-32}$ Three of these cases were transfusion-associated. Fever was a predominant symptom in 7 of these cases; this was not the case in that of a premature infant with transfusionassociated disease. In all published cases, infection was directly confirmed by blood smear examination, direct amplification of pathogen DNA, or by inoculation of a laboratory animal. One additional subject from Australia with no history of travel was reported to be positive by PCR for B. duncani. ${ }^{33}$ His clinical presentation was not described in this publication.

Seropositivity to $B$. duncani appears to be common in asymptomatic individuals. In northern California, 3.5\% of all individuals and $16 \%$ of higher-risk subjects were seropositive. This was corroborated by a separate study from northern California showing a seroprevalence of $17.8 \% .^{34}$ Finally, a private reference laboratory reported that $27 \%$ of clinical specimens and $2 \%$ of specimens from prospective blood donors had titers to $B$. duncani of at least $1: 256$. $^{35}$

In no published report was $B$. duncani directly detected in afebrile patients who lacked other objective clinical or laboratory signs of disease.

\section{Search 4: Evidence of Tick-borne Human Bartonella Infection}

A total of 200 articles was identified, the great majority of them reporting the detection of Bartonella within ticks. Nine articles were reviewed further for direct evidence of human Bartonella infection transmitted by a tick bite, or the vector competence of ticks to transmit Bartonella spp. to a host. The most direct evidence of tick-borne human bartonellosis comes from a study of 3 patients from southern France investigating the "scalp eschar and neck lymphadenopathy after tick bite" syndrome. ${ }^{36}$ The eschars from 2 of these patients were positive by PCR for $B$. henselae. These patients, however, did not have an identified tick bite, and had other risk factors for Bartonella infection (including cat exposure). A third patient had an eschar that was negative by PCR for B. henselae. He did, however, provide an ornate sheep tick, Dermacentor marginatus, that was retrieved from the site of the eschar; this tick was positive for
B. henselae. Our search did not yield other articles demonstrating tick transmission of Bartonella to humans. Three studies have demonstrated transmission of Bartonella spp. by ticks using artificial feeding systems and murine transmission models. One study demonstrated that the brown dog tick, Rhipicephalus sanguineus, could become infected with $B$. vinsonii subsp. berkhoffii when feeding using a capillary tube system. ${ }^{37}$ A second study found that I. ricinus ticks could acquire $B$. henselae after feeding on infected blood using a membrane feeding system. Neither of these studies demonstrated transmission of the organism from the tick to a mammalian host. The only study to do so found that $B$. birtlesii could be transmitted to mice by I. ricinus. ${ }^{37-39}$ This study has not been corroborated by evidence that transmission occurs in nature. No study has yet investigated transmission of $B$. henselae by I. scapularis.

\section{Search 5: Evidence of Simultaneous Lyme Disease and Bartonella spp. Infection}

This search yielded 155 articles, of which 8 were appropriate for further review based on the criteria described in the Methods section. Three of these publications presented patients with putative Bartonella/Lyme disease coinfection. ${ }^{40}$ One patient had several months of nonspecific symptoms, then sudden vision loss that was attributed to neuroretinitis. Titers were strongly positive to $B$. henselae $(>1: 1024)$. The patient had detectable peripheral and cerebrospinal fluid IgM antibodies to B. burgdorferi, but had a negative $B$. burgdorferi $\mathrm{IgG}$ by established interpretive criteria. The second publication reported 4 symptomatic patients in whom DNA from both $B$. henselae and B. burgdorferi were found in the cerebrospinal fluid. ${ }^{41}$ Only one of these subjects was seropositive to B. burgdorferi. Very little clinical information was given about these patients, including whether there was cerebrospinal fluid evidence of meningitis. Amplicons from PCR reactions were not sequenced. Finally, a third publication reported testing results from 2 patients from Poland with meningitis; no further clinical details were provided in the study. ${ }^{42}$ Of these patients, one had $B$. henselae DNA in the cerebrospinal fluid; this individual was seronegative for antibodies to $B$. henselae, but had detectable $\operatorname{IgG}$ antibodies to $B$. burgdorferi. A second patient was found to have equivocal levels of antibodies to $B$. henselae and equivocal levels of IgM antibodies to B. burgdorferi. Serologic evaluation for Lyme disease in this study did not correspond to current recommendations for 2-tier testing.

A number of other studies have suggested that occupationally exposed individuals are frequently seropositive for antibodies to both B. burgdorferi and Bartonella. A serosurvey of at-risk individuals in Lublin, Poland (forestry workers and farmers) found that $8.9 \%$ of individuals had antibodies to both Bartonella spp. and B. burgdorferi. ${ }^{43} \mathrm{~A}$ separate study from the Warsaw region found seropositivity to both organisms in $10 \%$ of forestry workers. ${ }^{44}$ A study of patients with a variety of rheumatic disease manifestations 
from a Lyme disease-hyperendemic region found antibodies to Bartonella in $62 \%$ of subjects and direct detection of the organism in $41.1 \%$; none of these patients, however, had documentation of Lyme disease. ${ }^{45}$ Finally, in an Australian study, 2 patients were described as having evidence of simultaneous Bartonella infection and Lyme disease. The clinical syndromes from these patients were not described; their seropositivity to Bartonella was an isolated IgM titer of $1: 40$, and these subjects only had IgM seroreactivity to B. burgdorferi. ${ }^{33}$

\section{DISCUSSION}

There is no debate in the scientific community that Ixodes spp. ticks transmit a number of important human pathogens, and sometimes in combination. In addition to B. burgdorferi, the causative agent of Lyme disease, Ixodes ticks may transmit B. microti and other human Babesia species, A. phagocytophilum, tick-borne encephalitis virus, Powassan virus, and emerging pathogens such as Borrelia miyamotoi. These infections may occur in isolation or in various combinations, and it is well established that coinfections have important clinical, diagnostic, and therapeutic implications. Active infection is characterized by objective clinical findings (eg, fever or laboratory abnormalities). Practitioners who frequently offer the diagnosis of chronic Lyme disease often do not rely on more accepted standards of clinical and laboratory testing. In such circumstances, many patients also receive spurious diagnoses of chronic anaplasmosis, babesiosis, and bartonellosis.

We have performed a systematic review of the medical literature in order to evaluate whether published science supports chronic, cryptic infections with these pathogens. Because of basic biological, clinical, and epidemiologic differences among HGA, babesiosis, and bartonellosis, different search terms were required for each pathogen.

A. phagocytophilum, the causative agent of HGA, is a rickettsial organism that produces an acute febrile systemic illness within about 2 weeks of an infectious tick bite. Infection is characterized by fever, constitutional symptoms, and laboratory abnormalities such as leukopenia, thrombocytopenia, and elevated levels of hepatic transaminases. Although HGA is potentially fatal, the infection will be selflimiting in survivors regardless of whether they are treated. As HGA is an infection of circulating leukocytes, both blood film examination and PCR of the blood can establish the presence of infection. Our search did not yield any reports of chronic, relapsing, or refractory HGA in humans. Persistent infection in domestic and wild ruminants, and persistent veterinary infections with related microorganisms (eg, Anaplasma marginale) cannot be assumed to predict the plausibility of chronic HGA in humans. To date there is no basis upon which to diagnose a human patient with chronic HGA.

Babesiosis is a malaria-like protozoan infection of erythrocytes that is transmitted by Ixodes spp. ticks. It may also be acquired from blood transfusions. Several species of
Babesia are capable of causing human disease; the most important of these are B. microti in the Northeastern and Midwestern US and B. divergens in Europe. Lyme-Babesia coinfection has been well established and may result in greater disease severity. ${ }^{10}$ Clinical babesiosis is nearly always dominated by fever and characteristic laboratory abnormalities, and the infection can be proved by direct visualization of the parasite on blood smear or detection of its DNA by blood PCR.

Relapsing or persistent infection can occur in immunocompromised patients, particularly those with lymphoma who are asplenic and received treatment with rituximab. Persistent babesiosis produces the same clinical and laboratory abnormalities that are seen in acute babesiosis, and patients remain both PCR and blood smear positive. In fact, immunocompromised patients who are at risk of persistent or recurrent babesiosis often have higher parasitemias and generally more severe disease. This is the only group of patients for whom there is evidence that a course of antibabesia drug therapy that exceeds 10 days duration is beneficial. ${ }^{21}$ We found no evidence that active babesiosis, as demonstrated by a positive PCR or blood smear, produces purely subjective complaints (eg, fatigue, pain, cognitive symptoms) that are unaccompanied by fever or by laboratory abnormalities. Asymptomatic blood donors have been the index cases for transfusion-associated babesiosis, so it may be the case that patent infection can actually be subclinical or nonspecific. If PCR-negative patients with purely subjective symptoms due to babesiosis exist, there are no published data on whether antibabesia therapy might be beneficial for them. The current standard of care is to treat only those individuals who can be shown by direct molecular or microscopic testing to have active babesiosis. ${ }^{46}$ Seroprevalence to $B$. microti clearly exceeds the incidence of clinically evident infections, suggesting that many individuals experience subclinical and asymptomatic infections. Thus, reliance on serology in the absence of direct demonstration of the organism could lead to erroneously attributing coincident symptoms to active infection.

This is particularly true for $B$. duncani, a pathogen responsible for a small number of human cases in the Pacific Northwest. Like other human babesias, $B$. duncani produces fever and hemolysis. Among the limited case reports there was no evidence of cryptic infection resulting only in subjective complaints. The high rates of background seropositivity to $B$. duncani, including in supposedly nonendemic areas according to one report, raise the question of whether there are cross-reactive antibodies in the population at large. This underscores the importance of directly demonstrating intraerythrocytic infection when pursuing a diagnosis of active babesiosis.

Unlike HGA and babesiosis, which in nature are exclusively transmitted to humans by Ixodes spp. ticks, we have found no convincing evidence that this is a natural or even plausible mode of transmission for Bartonella spp. Our search yielded no case in which tick-borne bartonellosis was 
unequivocally established. Not only is tick-borne human bartonellosis unfounded to date, but there is very little literature to support Lyme disease-Bartonella coinfection at all, regardless of the means of acquisition. Moreover, appropriate seroepidemiologic studies have not even been attempted in Lyme disease patients in the US to evaluate the seroprevalence of $B$. henselae in such individuals. While several small case series and reports in the literature purport to describe simultaneous Lyme disease and Bartonella infection, in no case did the laboratory corroboration of Lyme disease correspond to established diagnostic standards.

The putative association between ticks, Lyme disease, and $B$. henselae infection is ultimately derived from 2 problematic sources of data. The first is a limited number of reports of mostly European subjects in whom clinical infection with $B$. henselae and B. quintana has been temporally associated with a tick bite. ${ }^{47-49}$ The second source of data is the observation that many tick specimens contain Bartonella DNA when subjected to PCR analysis. ${ }^{50-54}$ This has been demonstrated primarily in the Eurasian ticks I. ricinus and I. persulcatus, and to a lesser degree, in the North American tick I. scapularis. Nonetheless, it should come as no surprise that ticks would contain Bartonella DNA - ticks feed on a variety of mammalian hosts that may be reservoirs for Bartonella spp. The presence of Bartonella DNA in the tick does not prove that the tick is a competent vector for transmission to a second mammalian host. Vector competence of I. scapularis ticks for B. henselae has never been demonstrated in an animal system.

\section{CONCLUSION}

The Ixodes spp. ticks that transmit B. burgdorferi are capable vectors of several human pathogens. In all cases, however, these infections produce defined clinical syndromes that are corroborated by objective clinical and laboratory findings. This is true for well-established Babesia-Lyme and Anaplasma-Lyme coinfections. Treatment and diagnosis of chronic coinfections, however, is clearly not justifiable in the absence of convincing objective evidence that these infections are present and active.

\section{References}

1. Centers for Disease Control and Prevention. Reported cases of Lyme disease by state or locality, 2002-2011. Available at: http://www.cdc. gov/lyme/stats/chartstables/reportedcases_statelocality.html. Accessed August 1, 2013.

2. Rabinstein A, Tikhomirov V, Kaluta A, Gelfmann N, Iannini P, Edwards L. Recurrent and prolonged fever in asplenic patients with human granulocytic ehrlichiosis. QJM. 2000;93(3):198-201.

3. Trofe J, Reddy KS, Stratta RJ, et al. Human granulocytic ehrlichiosis in pancreas transplant recipients. Transpl Infect Dis. 2001;3(1):34-39.

4. Miller LH, Neva FA, Gill F. Failure of chloroquine in human babesiosis (Babesia microti): case report and chemotherapeutic trials in hamsters. Ann Intern Med. 1978;88(2):200-202.

5. Ortiz JM, Eagle RC Jr. Ocular findings in human babesiosis (Nantucket fever). Am J Ophthalmol. 1982;93(3):307-311.
6. Machtinger L, Telford SR 3rd, Inducil C, Klapper E, Pepkowitz SH, Goldfinger D. Treatment of babesiosis by red blood cell exchange in an HIV-positive, splenectomized patient. J Clin Apher. 1993; 8(2):78-81.

7. Cahill KM. Babesiosis: unappreciated even in endemic areas. J Community Health. 1995;20(4):315-320.

8. Gupta P, Hurley RW, Helseth PH, Goodman JL, Hammerschmidt DE. Pancytopenia due to hemophagocytic syndrome as the presenting manifestation of babesiosis. Am J Hematol. 1995;50(1):60-62.

9. Falagas ME, Klempner MS. Babesiosis in patients with AIDS: a chronic infection presenting as fever of unknown origin. Clin Infect Dis. 1996;22(5):809-812.

10. Krause PJ, Telford SR 3rd, Spielman A, et al. Concurrent Lyme disease and babesiosis. Evidence for increased severity and duration of illness. JAMA. 1996;275(21):1657-1660.

11. Evenson DA, Perry E, Kloster B, Hurley R, Stroncek DF. Therapeutic apheresis for babesiosis. J Clin Apher. 1998;13(1):32-36.

12. Krause PJ, Spielman A, Telford SR 3rd, et al. Persistent parasitemia after acute babesiosis. N Engl J Med. 1998;339(3):160-165.

13. White DJ, Talarico J, Chang HG, Birkhead GS, Heimberger T, Morse DL. Human babesiosis in New York State: review of 139 hospitalized cases and analysis of prognostic factors. Arch Intern Med. 1998;158(19):2149-2154.

14. Setty S, Khalil Z, Schori P, Azar M, Ferrieri P. Babesiosis. Two atypical cases from Minnesota and a review. Am J Clin Pathol. 2003;120(4):554-559.

15. Wudhikarn K, Perry EH, Kemperman M, Jensen KA, Kline SE. Transfusion-transmitted babesiosis in an immunocompromised patient: a case report and review. Am J Med. 2011;124(9):800-805.

16. El-Bahnasawy MM, Khalil HH, Morsy TA. Babesiosis in an Egyptian boy aquired from pet dog, and a general review. J Egypt Soc Parasitol. 2011;41(1):99-108.

17. Lubin AS, Snydman DR, Miller KB. Persistent babesiosis in a stem cell transplant recipient. Leuk Res. 2011;35(6):e77-e78.

18. Herman JH, Ayache S, Olkowska D. Autoimmunity in transfusion babesiosis: a spectrum of clinical presentations. J Clin Apher. 2010;25(6):358-361.

19. Wormser GP, Prasad A, Neuhaus E, et al. Emergence of resistance to azithromycin-atovaquone in immunocompromised patients with Babesia microti infection. Clin Infect Dis. 2010;50(3):381-386.

20. Blue D, Graves V, McCarthy L, Cruz J, Gregurek S, Smith D. Fatal transfusion-transmitted Babesia microti in the Midwest. Transfusion. 2009;49(1):8.

21. Krause PJ, Gewurz BE, Hill D, et al. Persistent and relapsing babesiosis in immunocompromised patients. Clin Infect Dis. 2008;46(3): 370-376.

22. Clark IA, Budd AC, Hsue G, et al. Absence of erythrocyte sequestration in a case of babesiosis in a splenectomized human patient. Malar J. 2006;5:69.

23. Stowell CP, Gelfand JA, Shepard JA, Kratz A. Case records of the Massachusetts General Hospital. Case 17-2007. A 25-year-old woman with relapsing fevers and recent onset of dyspnea. $N$ Engl $J$ Med. 2007;356(22):2313-2319.

24. MacDonald KL, Osterholm MT, LeDell KH, et al. A case-control study to assess possible triggers and cofactors in chronic fatigue syndrome. Am J Med. 1996;100(5):548-554.

25. Sherr VT. Panic attacks may reveal previously unsuspected chronic disseminated lyme disease. J Psychiatr Pract. 2000;6(6):352-356.

26. Bloch EM, Herwaldt BL, Leiby DA, et al. The third described case of transfusion-transmitted Babesia duncani. Transfusion. 2012;52(7): $1517-1522$.

27. Herwaldt BL, Kjemtrup AM, Conrad PA, et al. Transfusion-transmitted babesiosis in Washington State: first reported case caused by a WA1type parasite. J Infect Dis. 1997;175(5):1259-1262.

28. Herwaldt BL, Linden JV, Bosserman E, Young C, Olkowska C, Wilson M. Transfusion-associated babesiosis in the United States: a description of cases. Ann Intern Med. 2011;155(8):509-519. 
29. Kjemtrup AM, Lee B, Fritz CL, Evans C, Chervenak M, Conrad PA. Investigation of transfusion transmission of a WA1-type babesial parasite to a premature infant in California. Transfusion. 2002;42(11): 1482-1487.

30. Persing DH, Herwaldt BL, Glaser C, et al. Infection with a babesia-like organism in northern California. N Engl J Med. 1995;332(5):298-303.

31. Quick RE, Herwaldt BL, Thomford JW, et al. Babesiosis in Washington State: a new species of Babesia? Ann Intern Med. 1993;119(4):284-290.

32. Thomford JW, Conrad PA, Telford SR 3rd, Mathiesen D, et al. Cultivation and phylogenetic characterization of a newly recognized human pathogenic protozoan. J Infect Dis. 1994;169(5):1050-1056.

33. Mayne PJ. Emerging incidence of Lyme borreliosis, babesiosis, bartonellosis, and granulocytic ehrlichiosis in Australia. Int J Gen Med. 2011;4:845-852.

34. Fritz CL, Kjemtrup AM, Conrad PA, et al. Seroepidemiology of emerging tickborne infectious diseases in a Northern California community. J Infect Dis. 1997;175(6):1432-1439.

35. Prince HE, Lape-Nixon M, Patel H, Yeh C. Comparison of the Babesia duncani (WA1) IgG detection rates among clinical sera submitted to a reference laboratory for WA1 $\mathrm{IgG}$ testing and blood donor specimens from diverse geographic areas of the United States. Clin Vaccine Immunol. 2010;17(11):1729-1733.

36. Angelakis E, Pulcini C, Waton J, et al. Scalp eschar and neck lymphadenopathy caused by Bartonella henselae after Tick Bite. Clin Infect Dis. 2010;50(4):549-551.

37. Billeter SA, Kasten RW, Killmaster LF, et al. Experimental infection by capillary tube feeding of Rhipicephalus sanguineus with Bartonella vinsonii subspecies berkhoffii. Comp Immunol Microbiol Infect Dis. 2012;35(1):9-15.

38. Cotte V, Bonnet S, Le Rhun D, et al. Transmission of Bartonella henselae by Ixodes ricinus. Emerg Infect Dis. 2008;14(7):1074-1080.

39. Reis C, Cote M, Le Rhun D, et al. Vector competence of the tick Ixodes ricinus for transmission of Bartonella birtlesii. PLoS Negl Trop Dis. 2011;5(5):e1186.

40. Gupta PK, Patel R, Bhatti MT. Neuroretinitis secondary to concurrent infection with cat scratch disease and lyme disease. Eye. 2009; 23(7):1607.

41. Eskow E, Rao RV, Mordechai E. Concurrent infection of the central nervous system by Borrelia burgdorferi and Bartonella henselae: evidence for a novel tick-borne disease complex. Arch Neurol. 2001;58(9):1357-1363.

42. Podsiadly E, Chmielewski T, Tylewska-Wierzbanowska S. Bartonella henselae and Borrelia burgdorferi infections of the central nervous system. Ann N Y Acad Sci. 2003;990:404-406.

43. Chmielewska-Badora J, Moniuszko A, Zukiewicz-Sobczak W, Zwolinski J, Platek J, Pancewicz S. Serological survey in persons occupationally exposed to tick-borne pathogens in cases of coinfections with Borrelia burgdorferi, Anaplasma phagocytophilum, Bartonella spp. and Babesia microti. Ann Agric Environ Med. 2012;19(2):271-274.

44. Podsiadly E, Chmielewski T, Karbowiak G, Kedra E, TylewskaWierzbanowska S. The occurrence of spotted fever rickettsioses and other tick-borne infections in forest workers in Poland. Vector Borne Zoonotic Dis. 2011;11(7):985-989.

45. Maggi RG, Mozayeni BR, Pultorak EL, et al. Bartonella spp. bacteremia and rheumatic symptoms in patients from Lyme disease-endemic region. Emerg Infect Dis. 2012;18(5):783-791.

46. Wormser GP, Dattwyler RJ, Shapiro ED, et al. The clinical assessment, treatment, and prevention of lyme disease, human granulocytic anaplasmosis, and babesiosis: clinical practice guidelines by the Infectious Diseases Society of America. Clin Infect Dis. 2006;43(9): 1089-1134.

47. Lucey D, Dolan MJ, Moss CW, et al. Relapsing illness due to Rochalimaea henselae in immunocompetent hosts: implication for therapy and new epidemiological associations. Clin Infect Dis. 1992;14(3):683-688.

48. Arnez M, Luznik-Bufon T, Avsic-Zupanc T, et al. Causes of febrile illnesses after a tick bite in Slovenian children. Pediatr Infect Dis J. 2003;22(12):1078-1083.

49. Morozova OV, Chernousova N, Morozov IV. Detection of the Bartonella DNA by the method of nested PCR in patients after tick bites in Novosibirsk region [Russian]. Mol Gen Mikrobiol Virusol 2005;(4):14-17.

50. Sytykiewicz H, Karbowiak G, Werszko J, Czerniewicz P, Sprawka I, Mitrus J. Molecular screening for Bartonella henselae and Borrelia burgdorferi sensu lato co-existence within Ixodes ricinus populations in central and eastern parts of Poland. Ann Agric Environ Med. 2012;19(3):451-456.

51. Dietrich F, Schmidgen T, Maggi RG, et al. Prevalence of Bartonella henselae and Borrelia burgdorferi sensu lato DNA in ixodes ricinus ticks in Europe. Appl Environ Microbiol. 2010;76(5):1395-1398.

52. Morozova OV, Cabello FC, Dobrotvorsky AK. Semi-nested PCR detection of Bartonella henselae in Ixodes persulcatus ticks from Western Siberia, Russia. Vector Borne Zoonotic Dis. 2004;4(4): 306-309.

53. Sanogo YO, Zeaiter Z, Caruso G, et al. Bartonella henselae in Ixodes ricinus ticks (Acari: Ixodida) removed from humans, Belluno province, Italy. Emerg Infect Dis. 2003;9(3):329-332.

54. Adelson ME, Rao RV, Tilton RC, Cabets K, et al. Prevalence of Borrelia burgdorferi, Bartonella spp., Babesia microti, and Anaplasma phagocytophila in Ixodes scapularis ticks collected in Northern New Jersey. J Clin Microbiol. 2004;42(6):2799-2801. 